\title{
Condensation transition and ensemble inequivalence in the Discrete Nonlinear Schrödinger Equation
}

\author{
Giacomo Gradenigo, ${ }^{1}$ Stefano Iubini, ${ }^{2,3}$ Roberto Livi, ${ }^{4,3,2}$ and Satya N. Majumdar ${ }^{5}$ \\ ${ }^{1}$ Gran Sasso Science Institute, Viale F. Crispi 7, 67100 L'Aquila, Italy ${ }^{*}$ \\ ${ }^{2}$ Consiglio Nazionale delle Ricerche, Istituto dei Sistemi Complessi, \\ via Madonna del Piano 10, I-50019 Sesto Fiorentino, Italy \\ ${ }^{3}$ Istituto Nazionale di Fisica Nucleare, Sezione di Firenze, \\ via G. Sansone 1 I-50019, Sesto Fiorentino, Italy \\ ${ }^{4}$ Dipartimento di Fisica e Astronomia and CSDC, Università di Firenze, \\ via G. Sansone 1 I-50019, Sesto Fiorentino, Italy \\ ${ }^{5}$ LPTMS, CNRS, Université Paris-Sud, Université Paris-Saclay, 91405 Orsay, France
}

\begin{abstract}
The thermodynamics of the discrete nonlinear Schrödinger equation in the vicinity of infinite temperature is explicitly solved in the microcanonical ensemble by means of large-deviation techniques. A first-order phase transition between a thermalized phase and a condensed (localized) one occurs at the infinite-temperature line. Inequivalence between statistical ensembles characterizes the condensed phase, where the grand-canonical representation does not apply. The control over finite size corrections of the microcanonical partition function allows to design an experimental test of delocalized negative-temperature states in lattices of cold atoms.
\end{abstract}

\section{INTRODUCTION}

The Discrete Nonlinear Schrödinger equation (DNLSE) [1] is a very useful phenomenological model for various physical phenomena, e.g. light propagating in arrays of optical waveguides [2] and Bose-Einstein condensates in optical lattices [3, 4. In fact, it can be viewed as a lattice version of the Gross-Pitaevskii equation (nonlinear Schrödinger equation), i.e. the semiclassical model for the bosonic condensate wavefunction [5]. In this paper, we solve the statistical mechanics of this model close to infinite temperature, where it exhibits a peculiar first-order phase transition from a thermalized phase to a localized/condensed one. So far, the condensation phenomenon in the DNLSE has been investigated in depth only numerically [4, 6] 8 . It occurs when a macroscopic fraction of energy is localized on one/few lattice sites and it can be traced back to the existence of two conserved quantities [9, 26]. As we are going to make clear in what follows, a grand-canonical description of this condensation transition is not allowed, because of the inequivalence of statistical ensembles. The overall method for estimating the microcanonical partition function in the vicinity of the phase transition relies on well established large-deviation techniques. Moreover, our results apply in any spatial dimension.

Before entering into technical details, we want to point out that our contribution can be cast in the more general frame of a deeper understanding of the mechanisms responsible for wave-function localization in systems of interacting quantum particles. This is one among the most exciting open problems nowadays, both in condensed matter and in statistical physics [10 12]. As opposed to the well known phenomenon of Many-Body Localization,

\footnotetext{
* giacomo.gradenigo@gssi.it
}

taking place when interactions are weakly non-linear, we show here that localization in the DNLSE is essentially due to strong nonlinear effects. Notice that this phenomenon is not due to the proximity to a standard integrable system, e.g. [13, 14, as usually invoked for many non-linear models, nor it can be attributed to the effect of disorder, as in most models of glasses. Here we are rather facing and characterizing quite a unique and unprecedented scenario: lack of energy equipartition, driven by genuine strong nonlinearities.

\section{MODEL AND STATE OF THE ART}

The one-dimensional parameter-less DNLSE Hamiltonian is defined in terms of a scalar complex field $z_{j}$, taking values on a lattice of $N$ sites with periodic boundary conditions:

$$
\mathcal{H}=\sum_{j=1}^{N}\left(z_{j}^{*} z_{j+1}+z_{j} z_{j+1}^{*}\right)+\sum_{j=1}^{N}\left|z_{j}\right|^{4},
$$

where quadratic terms represent standard hopping energies between nearest-neighbour sites, while non-linear terms represent an on-site potential coming from the repulsive contact energy in the Gross-Pitaevskii equation. The corresponding equations of motion are

$$
i \dot{z}_{j}=-\frac{\partial \mathcal{H}}{\partial z_{j}^{*}}=-\left(z_{j+1}+z_{j-1}\right)-2\left|z_{j}\right|^{2} z_{j} .
$$

One can easily check that this dynamics conserves $\mathcal{H}$ and the total mass $\mathcal{A}=\sum_{j=1}^{N}\left|z_{j}\right|^{2}$. We denote by $E$ and $A$ the real values taken by the observables $\mathcal{H}$ and $\mathcal{A}$, respectively.

The thermodynamic behaviour of the DNLSE depends on the values taken by these two parameters or by their averages, depending on whether the system is isolated 


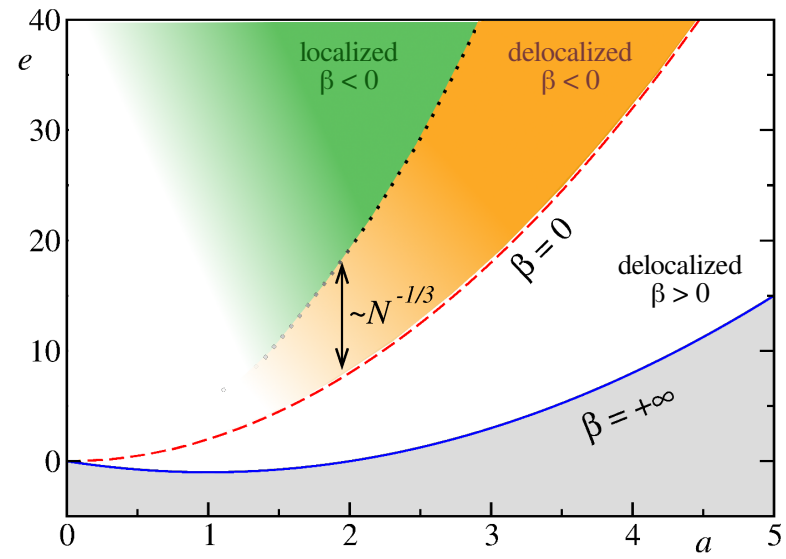

Figure 1. Equilibrium microcanonical phase diagram of the DNLSE in the $(a, e)$-plane. Blue solid and red dashed lines correspond to the ground state and the infinite temperature isothermal $(\beta=0)$, respectively. Localized (green) and delocalized negative-temperature (orange) regions are drawn for a chain of $N=100$ lattice sites. Black dots identify the line of critical energy densities, see text.

(microcanonical conditions) or interacting with an external reservoir (grand-canonical conditions). Accordingly, it is expected that the two conserved quantities can be mapped to a couple of values of temperature $T$ and chemical potential $\mu$.

The main thermodynamic features of this model (e.g., see 15) are summarized in the phase diagram shown in Fig. 1. where $e=E / N$ and $a=A / N$ are the energy and mass densities, respectively.

In [15] it was shown within the grand-canonical ensemble that the condition $e=2 a^{2}$ identifies the infinitetemperature line $\left(\beta=1 / T=0\right.$ and $\left.\mu^{-1}=0\right)$, while the ground state line $(\beta=+\infty)$ is identified by the relation $e=a^{2}-2 a$ and $\mu=2(a-1)$. Below this line no physical state is allowed, while a standard thermal phase extends in between these two lines: dynamics (2) makes any initial state eventually thermalize to energy equipartition. Above the $\beta=0$ line numerical studies (e.g., see [4, 6]) have shown that the hamiltonian dynamics of the DNLSE exhibits localized breather excitations. Such excitations can survive over extremely long times (exponentially long in their mass 6]) even in a chain made of a few tens of sites [4. The coupling of the localized breathers among themselves, through the background energy fluctuations, is very weak and their coalescence into a single localized giant breather, even if possible (in fact, breathers are known to merge when they collide 4), is practically unobservable. This process can be dramatically accelerated by replacing the hamiltonian dynamics with a stochastic evolution rule, conserving both $\mathcal{H}$ and $\mathcal{A}$. As shown in [17, 18] this stochastic dynamics yields full localization as a product of a coarsening process.

In Ref. [15] it was also conjectured that the region above the $\beta=0$ line should correspond to a negativetemperature phase. A numerical evidence of this conjec- ture was presented in [7, 8, 16, although no conclusive theoretical argument for the existence of this phase has been presented so far. On the other hand, several authors, on the basis of general thermodynamic arguments, have argued that this negative-temperature phase should not be a genuine thermodynamic equilibrium one (e.g., see [19 25]). In this paper we provide an answer to all of these open questions, by computing explicitly the microcanonical partition function of the DNLSE close to the $\beta=0$ line for large $N$, using large-deviation techniques similar to those employed in $26+29$. In particular, the study of the microcanonical entropy here presented closely follows the large deviations study of runand-tumble particles in 28, though the context of the latter was entirely different.

\section{STATISTICAL MECHANICS OF THE DNLSE}

We start by pointing out that close to the infinite temperature line the scalar complex fields in their polar representation, $z_{j}=\rho_{j} e^{i \phi_{j}}$, have random phases, so that in the limit of large values of $N$ the bilinear hopping term in $\sqrt{1}$ is of order $\sqrt{N}$, being the sum of random sign numbers, while the nonlinear contribution to energy is of order $N$, being the sum of positive numbers. That is why at high temperature hopping terms yield a subleading contribution to energy and the $\beta=0$ line is determined by the relation $e=2 a^{2}$, even according to the exact calcultion of [15] where hopping terms are retained. The latter result really indicates that at high temperatures the total energy reduces to the contribution of the local quartic potential. In the following calculations, we will thus always neglect hopping terms. While a more thorough discussion on this random phase approximantion can be found in [8, 30, here it is worth anticipating one of our results, namely that in the limit $N \rightarrow \infty$ the microcanonical temperature diverges for all energies $e>2 a^{2}$. This furnishes an "à posteriori" argument to accept the random-phase approximantion as a reasonable one for the whole region $e>2 a^{2}$ of the phase diagram. We stress that the results hereafter reported hold for any value of $d$, since any relation with the spatial dimension $d$ enters this problem through a $d$-dimensional hopping term $\sum_{k=1}^{d}\left(z_{\vec{j}}^{*} z_{\vec{j}+\vec{e}_{k}}+z_{\vec{j}} z_{\vec{j}+\vec{e}_{k}}^{*}\right)$, where $\vec{j} \in \mathbb{Z}^{d}$ and $\left\{\vec{e}_{k}\right\}$ with $k=1, \cdots, d$ is the unit vector basis for $\mathbb{Z}^{d}$. To our knowledge this is the first theoretical model exhibiting a localization transition, induced by nonlinearity, in finite dimensions $d>1$.

Since $A$ and $E$ are the only two conserved quantities, the microcanonical partition function for $\beta \rightarrow 0$, where 
the hopping term can be neglected, reads:

$$
\Omega_{N}(A, E)=(2 \pi)^{N} \int \mathcal{D} \rho \delta\left(A-\sum_{j=1}^{N} \rho_{j}^{2}\right) \delta\left(E-\sum_{j=1}^{N} \rho_{j}^{4}\right),
$$

where we have used the short-hand notation $\int \mathcal{D} \rho=$ $\int_{0}^{\infty} \prod_{j=1}^{N} d \rho_{j} \rho_{j}$ and we have integrated over the phases $\phi_{j}$. It is convenient to compute first the Laplace transform $\tilde{\Omega}_{N}(\lambda, E)$ :

$$
\tilde{\Omega}_{N}(\lambda, E)=\int_{0}^{\infty} d A e^{-\lambda A} \Omega_{N}(A, E)
$$

By performing the change of variables $\rho_{j}^{4}=\varepsilon_{j}$, this can be rewritten as follows

$$
\tilde{\Omega}_{N}(\lambda, E)=\left(\frac{\pi}{\lambda}\right)^{N} \int \mathcal{D} \varepsilon f_{\lambda}\left(\varepsilon_{j}\right) \delta\left(E-\sum_{j=1}^{N} \varepsilon_{j}\right),
$$

with

$$
\int \mathcal{D} \varepsilon=\int_{0}^{\infty} \prod_{j=1}^{N} d \varepsilon_{j}
$$

and

$$
f_{\lambda}(\varepsilon)=\lambda /(2 \sqrt{\varepsilon}) \exp (-\lambda \sqrt{\varepsilon}) .
$$

The function $f_{\lambda}(\varepsilon)$ can be recognized as a normalized probability distribution with a stretched-exponential tail, with average and variance

$$
\langle\varepsilon\rangle=2 / \lambda^{2} \quad, \quad \sigma^{2}=\left\langle\varepsilon^{2}\right\rangle-\langle\varepsilon\rangle^{2}=20 / \lambda^{4} .
$$

The integral on the r.h.s. of (5) is formally identical to the probability that the sum of $N$ independent identically distributed random variables $\varepsilon_{j}$, with individual distribution $f_{\lambda}(\varepsilon)$, takes the value $E$. Large-deviation theory predicts that, due to the stretched exponential tail of $f_{\lambda}(\varepsilon)$, such probability undergoes a condensation phenomenon when $E$ overtakes the threshold value $E_{t h}=N\langle\varepsilon\rangle[26$ 28, 31, 32] : a finite fraction of the total energy localizes onto a single site in the thermodynamic limit. Let us stress that the exponent of the slow stretched-exponential decay of $f_{\lambda}(\varepsilon)$ is uniquely determined by the order of the non-linearity. For instance, if the non-linear term had been $\left|z_{j}\right|^{6}$, one would have obtained $f_{\lambda}(\varepsilon) \sim \exp \left(-\varepsilon^{1 / 3}\right)$.

Following a standard procedure 26 29, one should compute the Laplace transform of $\tilde{\Omega}_{N}(\lambda, E)$ with respect to $E$, i.e.

$$
\mathcal{Z}_{N}(\lambda, \beta)=\int_{0}^{\infty} d E e^{-\beta E} \tilde{\Omega}_{N}(\lambda, E),
$$

which can be rewitten in the following form

$$
\mathcal{Z}_{N}(\lambda, \beta)=(\pi / \lambda)^{N} \exp \{N \log [z(\lambda, \beta)]\},
$$

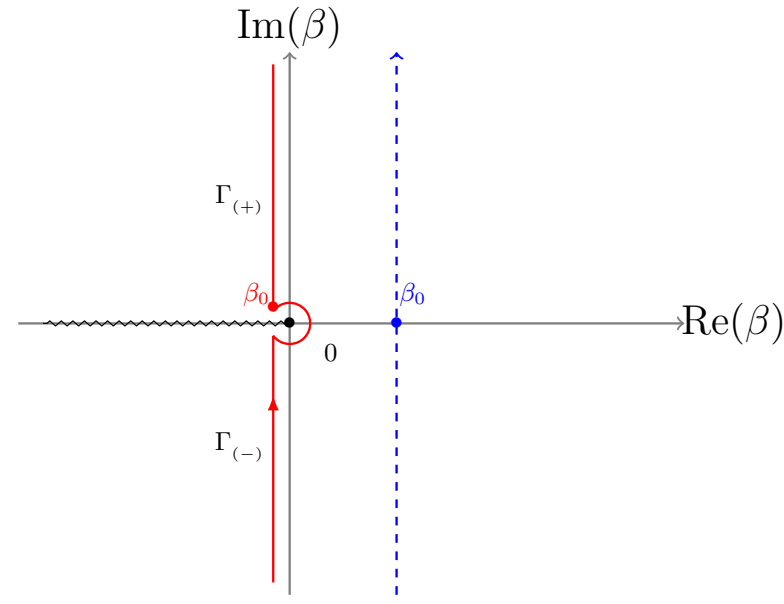

Figure 2. Analytic structure of $\mathcal{Z}_{N}(\lambda, \beta)$ in the complex $\beta$ plane. The microcanonical partition function can be computed by the Bromwich contour represented by the blue line and by the red line for $E<E_{t h}$ and $E>E_{t h}$, respectively.

where

$$
z(\lambda, \beta)=\frac{\lambda}{\sqrt{\beta}} e^{\lambda^{2} /(4 \beta)} \operatorname{Erfc}\left(\frac{\lambda}{2 \sqrt{\beta}}\right),
$$

and $\operatorname{Erfc}(x)=\int_{x}^{\infty} e^{-t^{2}} d t$ is the complementary error function. For any fixed $\lambda>0$, the expression in (11) can be taken as the definition of a function in the complex $\beta$ plane. In particular $\operatorname{Erfc}[\lambda /(2 \sqrt{\beta})]$, and accordingly $z(\lambda, \beta)$, has a branch-cut on the negative real semiaxis (see Fig. 2). Inverting formally this Laplace transform with respect to $\beta$, we obtain $\tilde{\Omega}_{N}(\lambda, E)$ as

$$
\tilde{\Omega}_{N}(\lambda, E)=\int_{\beta_{0}-i \infty}^{\beta_{0}+i \infty} \frac{d \beta}{2 \pi i} e^{\beta E} \mathcal{Z}_{N}(\lambda, \beta)
$$

For large $N$ the integral on the right hand side (r.h.s.) can be estimated by a saddle-point approximation at the real value $\beta_{0}$, which is the solution of the saddle-point equation

$$
E=-\frac{\partial \ln \mathcal{Z}_{N}(\lambda, \beta)}{\partial \beta}
$$

Taking into account the analytic properties of $\mathcal{Z}_{N}(\lambda, \beta)$ one finds that a real positive solution $\beta_{0}$ exists only if $E<E_{t h}$ and the integral in 112 can be performed over the vertical contour (dashed blue line in Fig 2 intersecting the positive real axis in $\beta_{0}$. In particular, this guarantees also the equivalence between the micro-canonical and the grand-canonical statistical ensembles. Conversely, for $E>E_{t h}$ a real solution of (13) does not exist, because the integration contour cannot intersect the branch-cut along the real negative semiaxis in the complex $\beta$-plane. Accordingly, there is no equivalence between statistical ensembles. This notwithstanding, the microcanonical partition function can still be computed, because the integral 
in 12 can be explicitly performed by following a suitably deformed contour (continuous red line in Fig 2). In practice, this demands the application of a large deviation technique, which amounts to estimate $\tilde{\Omega}_{N}(\lambda, E)$ at different orders of $E-E_{t h}$ as a power of $N$ : details can be found in 30]. Here we just summarize the main results. At the energy scale $E-E_{t h} \sim N^{\frac{1}{2}}$ (Gaussian regime), $\tilde{\Omega}_{N}(\lambda, E)$ can be estimated by expanding $\mathcal{Z}_{N}(\lambda, \beta)$ up to order $\beta \sim N^{-\frac{1}{2}}$. As an almost straightforward consequence of the central limit theorem one obtains [see also (8)]

$$
\tilde{\Omega}_{N}(\lambda, E)=\frac{1}{\sigma \sqrt{2 \pi N}} \exp \left[-\frac{\left(E-E_{\mathrm{th}}\right)^{2}}{2 \sigma^{2} N}\right] .
$$

In the regime of extreme large deviations the energy scale is $E-E_{t h} \sim N$ and $\mathcal{Z}_{N}(\lambda, \beta)$ has to be expanded up to order $\beta \sim N^{-1}$, yielding

$$
\tilde{\Omega}_{N}(\lambda, E) \sim \exp \left[-\sqrt{E-E_{\mathrm{th}}}\right]
$$

From previous studies on formally identical models [2628, we know that at the scale $E-E_{t h} \sim N^{1 / 2}$ there is no localized phase, while it is present when $E-E_{t h} \sim N$. The localization transition is expected to take place at the energy scale $E-E_{t h} \sim N^{\frac{2}{3}}$, where the analytic and the non-analytic contributions to the expansion of $z(\lambda, \beta)$ close to $\beta=0$ are of the same order 30 . In practice, this amounts to expand at the scale $\beta \sim N^{-\frac{1}{3}}$, thus yielding

$\tilde{\Omega}_{N}(\lambda, E)=\left(\frac{\pi}{\lambda}\right)^{N}\left[\frac{1}{\sigma \sqrt{2 \pi N}} \exp \left(-N^{1 / 3} \frac{\zeta^{2}}{2 \sigma^{2}}\right)+\mathcal{C}(\lambda, \zeta)\right]$

with

$$
\mathcal{C}(\lambda, \zeta)=N \sqrt{\frac{2 \pi}{\langle\varepsilon\rangle}} \exp \left[-N^{1 / 3} \chi(\zeta)\right] \quad, \quad \zeta=\frac{E-E_{\mathrm{th}}}{N^{2 / 3}}
$$

In the first term on the r.h.s. of 16 one can easily recognize the gaussian contribution in (14). The explicit expression of the function $\chi(\zeta)$ is quite involved (see Appendix $B$ in 30]). Here, we just report its asymptotic behaviours

$$
\chi(\zeta)=\left\{\begin{array}{l}
\frac{3}{2}\left(\frac{\sigma}{\langle\varepsilon\rangle}\right)^{2 / 3} \quad \zeta \rightarrow \zeta_{l} \\
\sqrt{\frac{2}{\langle\varepsilon\rangle} \sqrt{\zeta}-\frac{\sigma^{2}}{4\langle\varepsilon\rangle} \frac{1}{\zeta}+\mathcal{O}\left(\frac{1}{\zeta^{5 / 2}}\right), \quad \zeta \gg 1},
\end{array},\right.
$$

where

$$
\zeta_{l}=\frac{3}{2}\left(\frac{\sigma^{4}}{\langle\varepsilon\rangle}\right)^{1 / 3}
$$

is the spinodal point for the localized phase 30.

The final step for recovering an explicit expression of the microcanonical partition function amounts to com- puting the inverse Laplace-transform with respect to $\lambda$ :

$$
\begin{aligned}
& \Omega_{N}(A, E)=\frac{e^{N \log (\pi)}}{2 \pi i} \int_{\lambda_{0}-i \infty}^{\lambda_{0}+i \infty} d \lambda e^{N[a \lambda-\log (\lambda)]} \times \\
& {\left[e^{-N^{1 / 3} \chi(\zeta)}+e^{-N^{1 / 3} \zeta^{2} /\left(2 \sigma^{2}\right)}\right],}
\end{aligned}
$$

The leading contribution to this integral for large $N$ is given by the first exponential in the r.h.s., yielding the solution $\lambda_{0}=1 / a$ of the saddle-point equation

$$
\frac{\partial}{\partial \lambda}[a \lambda-\ln \lambda]=0 \quad \rightarrow \quad \lambda_{0}=\frac{1}{a}
$$

We finally obtain the expression

$\Omega_{N}(A, \zeta) \approx e^{N[1+\log (\pi a)]}\left[e^{-N^{1 / 3} \chi(\zeta)}+e^{-N^{1 / 3} \zeta^{2} /\left(2 \sigma^{2}\right)}\right]$

where $\sigma^{2}=20 a^{4}$ and $\langle\varepsilon\rangle=2 a^{2}$. The latter expression tells us that $E_{t h} / N=\langle\varepsilon\rangle=2 a^{2}$, i.e. the line at infinite temperature in Fig. 1, coincides with the energy density threshold, above which the theory of large deviations predicts the existence of the condensed phase 28.

In the limit of large values of $N$ we can thus write an estimate of the microcanonical entropy in the matching regime as follows

$$
S_{N}(a, \zeta)=N[1+\log (\pi a)]-N^{1 / 3} \Psi(\zeta)
$$

where

$$
\Psi(\zeta)=\inf _{\zeta}\left\{\chi(\zeta), \zeta^{2} /\left(2 \sigma^{2}\right)\right\}
$$

$S_{N}(a, \zeta)$ has an extensive part representing a background energy-independent entropy, while the subleading contribution contains information about the phase transition from a thermalized to a condensed (localized) phase. The critical value of $\zeta_{c}$ associated with the condensation transition is identified by the condition that the two contributions in Eq. 24) are equal, i.e. $\chi\left(\zeta_{c}\right)=\zeta_{c}^{2} /\left(2 \sigma^{2}\right)$, yielding $\zeta_{c}=2^{1 / 3} \zeta_{l}$ 30. The function $\Psi(\zeta)$ is shown in Fig. 3 and its derivative clearly exhibits a discontinuity at $\zeta_{c}$, thus providing a first-order transition mechanism at large $N$. In order to make more explicit its dependence on energy for values above the threshold, $E>E_{t h}$, the microcanonical entropy can be written as

$$
S_{N}(A, E)=N[1+\log (\pi A / N)]-N^{1 / 3} \Psi(E) .
$$

Clearly, any thermodynamic potential, computed taking derivatives of $S_{N}(A, E)$ with respect to energy, does vanish for $E>E_{t h}$ if we consider only the term of leading order in $N$ in Eq. 25). In such a regime any non trivial dependence on $E$ comes from the subleading term $N^{1 / 3} \Psi(E)$. It is for this reason that the discontinuity of $\Psi^{\prime}(E)$ at $E=E_{c}$ dominates the physics for energies above the threshold $E_{t h}$. In particular we have that the contribution $N[1+\log (\pi A / N)]$ to $S_{N}(A, E)$, in the regime $E>E_{t h}$, yields just a constant prefactor in front 


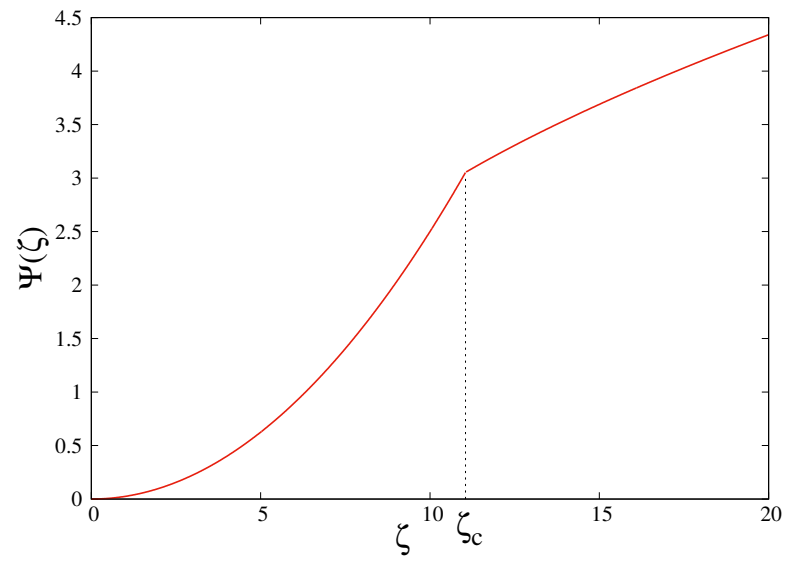

Figure 3. Singular part $\Psi(\zeta)$ of the microcanonical entropy, see $(23)$. Localization takes place at $\zeta_{c} \approx 11.05$, where $\Psi^{\prime}(\zeta)$ is discontinuous.

of the partition function, provided that one varies $E$ keeping $A$ fixed, a prefactor which simplifies in the expression of any microcanonical expectation value. We will in fact see that the participation ratio, which is defined as the average value of a certain function over the microcanonical measure, has a discontinuity at $E_{c}$.

Note that for finite $N$ one can identify a critical value of the energy, $E_{c}=E_{t h}+N^{2 / 3} \zeta_{c}$, which does not coincide with the threshold energy. The line of critical energy densities $E_{c}(a) / N$ is the black dotted line shown in the phase diagram of Fig. 1 for $N=100$. Even for moderately large system sizes, the condensation transition line is substantially shifted to higher energy densities with respect to the line $E_{t h}(a) / N$. The energy density gap $E_{c} / N-E_{t h} / N$ vanishes as $N^{-1 / 3}$ in the thermodynamic limit. Moreover, in the region above $E_{t h}$ the microcanonical inverse temperature

$$
\frac{1}{T}=\frac{\partial S_{N}(A, E)}{\partial E}=-\frac{1}{N^{1 / 3}} \Psi^{\prime}(E)
$$

is negative both above and below $E_{c}$. The difference between these two negative-temperature regions is characterized by the order parameter of localization, the socalled participation ratio:

$$
Y_{2}(E)=\left\langle\left(\sum_{j=1}^{N} \varepsilon_{j}^{2}\right) /\left(\sum_{j=1}^{N} \varepsilon_{j}\right)^{2}\right\rangle_{A, E}
$$

where the angular brackets $\langle\cdot\rangle_{A, E}$ denote the microcanonical equilibrium average. When energy is democratically distributed on all sites, i.e., $\varepsilon_{j} \sim E / N$ for every $j$, one has $Y_{2}(E) \sim 1 / N$. On the contrary, when a finite fraction of energy is localized on a single site, i.e., $\varepsilon_{j} \sim N$, then $Y_{2}(E) \sim 1$. As shown in 30] $Y_{2}(E)$, can be computed explicitly from 22 . For $E>E_{c}$ one obtains that $Y_{2}(E)$ is independent on $N$, thus identifying a localized phase. For $E<E_{c}$ one finds $Y_{2}(E) \sim 1 / N$, i.e., absence of localization.
Altogether, for finite sizes we obtain the following physical scenario: the line $E_{t h}$ separates the region at positive temperature, characterized by equivalence of ensembles and absence of localization from a region with negative microcanonical temperature with ensembles inequivalence. For $E_{t h}<E<E_{c}$ we identify a peculiar delocalized phase at negative temperature, (see the orange region in Fig. 1). For $E>E_{c}$ equilibrium states are localized and at negative temperature (green region in Fig. 11. This result suggests that one could design an experimental setup of cold-atoms in a finite optical lattice to explore the delocalized region above $E_{t h}$, where true negative-temperature states can be produced, while avoiding massive localization effects, that could destabilize the atomic condensate. Moreover, additional investigations should be devoted to a precise characterization of the delocalized negative-temperature region. In particular, it would be interesting to compare it with the weakly non-ergodic phase described in 33.

\section{FINAL REMARKS}

In the thermodynamic limit, the above scenario dramatically simplifies. In fact $E_{c} / N$ and $E_{t h} / N$ coincide, i.e. the delocalized negative-temperature region disappears and the temperature turns out to be infinite above $E_{t h} / N$. Moreover, the participation ratio vanishes continuously at $E_{t h} / N$. This notwithstanding, the microcanonical partition function guarantees that above $E_{t h} / N$ genuine thermodynamic equilibrium states at infinite temperature containing a unique localized breather do exist. Let us also remark that the exponent of the finite-size scaling correction $N^{1 / 3}$ of the microcanonical entropy [see [23] ] is uniquely, albeit non trivially, determined by the order of the non-linear interactions of the DNLSE. Accordingly, we have made explicit not only that localization in this model is essentially due to nonlinearity, but also that the order of the nonlinearity, which is a property of the microscopic interactions, directly affects a macroscopic property of the system, i.e. the finite-size scaling corrections of the entropy. Since experiments on bosonic condensates in optical lattices are typically performed for relatively small lattices 34, the model discussed in this paper indicates the possibility of novel and clear-cut predictions for laboratory tests.

\section{ACKNOWLEDGMENTS}

We thank for interesting discussions M. Baiesi, S. Franz, L. Leuzzi, G. Parisi, P. Politi, F. Ricci-Tersenghi, L. Salasnich, A. Scardicchio, F. Seno and A. Vulpiani. G.G. acknowledges the financial support of the Simons Foundation (Grant No. 454949, Giorgio Parisi) and the hospitality of "Sapienza", University of Rome, for the first stages of this work. S.I. acknowledges support from Progetto di Ricerca Dipartimentale BIRD173122/17 of 
the University of Padova. R.L. acknowledges partial support from project MIUR-PRIN2017 Coarse-grained de- scription for non- equilibrium systems and transport phenomena (CO- NEST) n. 201798CZL
[1] P. G. Kevrekidis, The Discrete Nonlinear Schrödinger Equation (Springer Verlag, Berlin, 2009).

[2] H.S. Eisenberg, Y. Silberberg, R. Morandotti, A.R. Boyd and J.S. Aitchison, Phys. Rev. Lett. 81, 3383 (1998).

[3] A. Trombettoni and A. Smerzi, Phys. Rev. Lett. 86, 2353 (2001).

[4] R. Franzosi, R. Livi, G.-L. Oppo and A. Politi, Nonlinearity 24, R89 (2011).

[5] L. Pitaevskii, S. Stringari, Bose-Einstein condensation and superfluidity, Oxford University Press.

[6] S. Iubini, L. Chirondojan, G.-L. Oppo, A. Politi and P. Politi, Phys. Rev. Lett. 122, 084102 (2019).

[7] R. Livi, R. Franzosi, and G.-L. Oppo, Phys. Rev. Lett. 97, 060401 (2006).

[8] S. Iubini, R. Franzosi, R. Livi, G.-L. Oppo and A. Politi, New Journal of Physics 15 , 023032 (2013).

[9] S. Chatterjee, Journal of Topology and Analysis, 9(04), 717 (2017).

[10] R. Nandkishore, D. A. Huse, Ann. Rev. Cond. Mat. Phys, 6 15-38 (2015).

[11] D. A. Abanin, Z. Papić, Ann. Phys. (Berlin) 529, No. 7, 1700169 (2017).

[12] E. Altman, Nature Phys. 14, 979-983 (2018).

[13] G. Benettin, H. Christodoulidi, A. Ponno, J. Stat. Phys. 152, 195-212 (2013).

[14] V. Ros, M. Müller, A. Scardicchio, Nuc. Phys. B 891, 420-465 (2015).

[15] K. O. Rasmussen, T. Cretegny, P.G. Kevrekidis and N. Gronbech-Jensen, Phys. Rev. Lett. 84, 3740 (2000)

[16] M. Johansson and K.O. Rasmussen, Phys. Rev. E 70, 066610 (2004).
[17] S. Iubini, A. Politi and P. Politi, J. Stat. Phys. 154, 1057-1073 (2014).

[18] S. Iubini, A. Politi and P. Politi, J. Stat. Mech. 073201 (2017).

[19] B. Rumpf and A.C. Newell, Phys. Rev. Lett. 87 , 054102 (2001).

[20] B. Rumpf, Phys. Rev. E 69 , 016618 (2004).

[21] B. Rumpf, Europhys. Lett. 78 , 26001 (2007).

[22] B. Rumpf, Phys. Rev. E 77 , 036606 (2008).

[23] B. Rumpf, Physica D 238 , 2067 (2009).

[24] J. Barré and L. Mangeolle, J. Stat. Mech. 043211 (2018).

[25] A. Y. Cherny, T. Engl and S. Flach, Phys. Rev. A 99, 023603 (2019).

[26] J. Szavits-Nossan, M. R. Evans and S. N. Majumdar, Phys. Rev. Lett. 112, 020602 (2014).

[27] J. Szavits-Nossan, M. R. Evans and S. N. Majumdar, J. Phys. A: Math. Theor. 47, 455004 (2014).

[28] G. Gradenigo and S. N. Majumdar, J. Stat. Mech. 053206 (2019).

[29] G. Gradenigo and E. Bertin, Entropy 19, 517 (2017).

[30] G. Gradenigo, S. Iubini, R. Livi and S. N. Majumdar, arXiv:1910.07461 [cond-mat.stat-mech] (2019).

[31] M. R. Evans and T. Hanney, J. Phys. A: Math. Gen. 38, R195 (2005).

[32] S.N. Majumdar, Les Houches lecture notes for the summer school "Exact Methods in Low-dimensional Statistical Physics and Quantum Computing" (Les Houches, July 2008), ed. by J. Jacobsen, S. Ouvry, V. Pasquier, D. Serban and L.F. Cugliandolo, Oxford University Press.

[33] T. Mithun, Y. Kati, C. Danieli, S. Flach Phys. Rev. Lett. 120, 184101 (2018).

[34] O. Morsch, M. Oberthaler, Rev. Mod. Phys. 78, 179 (2006) 